\title{
Rule Optimization of Self-organization Control of Traffic Signals in Urban Net Based on Hydrodynamic
}

\author{
Fusheng Zhong ${ }^{1, a}$ and Anlin Wang ${ }^{1}$ \\ ${ }^{1}$ Mechanical-electronic Engineering, Tongji University, CHINA
}

\begin{abstract}
Prior researchers indicate that hydrodynamics models of traffic-flow is lack of description of changing mechanism under urban traffic, and self-organization control system can not explain the dynamic characteristics of urban traffic flow clearly. The aim of the paper is to puts forward an optimized method on control rules that make the united application of hydrodynamics and self-organization system in signal control. The parameter sets of control rules are built from parameter sets of road network which are evolution under hydrodynamic equations such as the length of each lane, phase, queue length and so on .With the aim of the maximum traffic volume at each intersection in the road network, the control rules optimize its parameter sets to adapt to the dynamic change. By means of the computer simulation, the application of signal self-organizing control under hydrodynamic is proved effective in urban traffic. $^{\text {a }}$
\end{abstract}

\section{Introduction}

The urban traffic signal control system is a complex system as the change of traffic flow is complex.The equations are not accurate enough because of some unknown traffic mechanism, on the other hand the higher the degree of discretization is, the easier it is to deviate from the reality.Trafficflow under Hydrodynamics Models are commonly assumed to be continuous fluid and compressible, what makes it good to describe the collective behavior of vehicles. In fact, the interaction of vehicles, the judgement of the driver and other factors have great influence on the dynamic characteristics of traffic flow to make it discontinuous. Self-organizing control in urban traffic can easily linked the intersection to its interaction downstream and upstream which makes the control rules more reasonable, but if the parameter sets of rules are not sensitive to adapt to the change of traffic then the evolution of self-organizing are not determined.

Lighthill [1] defines the first order continuous module and Nagatani $\mathrm{T}$ [2] defines the discrete module with cellular automata(CA),both are research basis in their area. Most researchers focus on the how the traffic flow varies propagating disturbance in continuous mode or take more factors such as driver's behavior into consideration to modify the process during phase transition in discrete mode. XUE [3] proposes a two-time-scale equation to distinguish different relaxation time in speed propagating. GUPTA[4] takes driver's prospect into consideration to find out that reduces traffic jam efficiently. Biham [5] defines the traffic-flow models in two dimensions that explain how traffic flow change from Low density to high density under self-organization. Debashish [6] takes two typical CA

\footnotetext{
${ }^{\mathrm{a}}$ Corresponding author : ttxhway@126.com
} 
module to show that how traffic become jam and point out the progress depends on the linked signal cycle and phase difference. WEI [7] proposes a simple CA module to study the relations between intersections and regarded the intersection as the cell. The aim of this paper is to find more efficiently parameter sets of self-organization signal control, to use Hydrodynamics Models to enhance the description of the dynamics characteristics of the traffic flow. We put forward a new CA module which the intersections are the cell for control equation and lanes are also the cell for traffic flow's transfer. We also build the flow distribution module to modify the parameter sets more reasonable.

\section{Road-net and parameters}

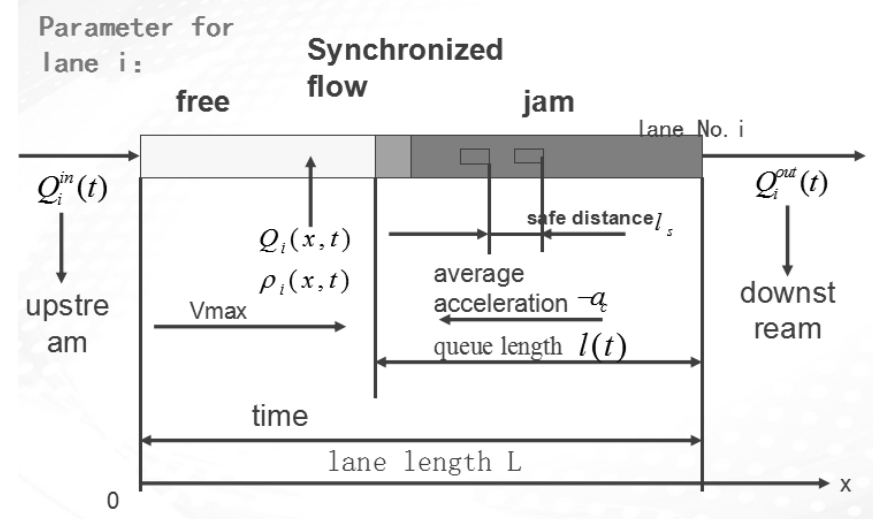

Figure 1. LANE MODULE

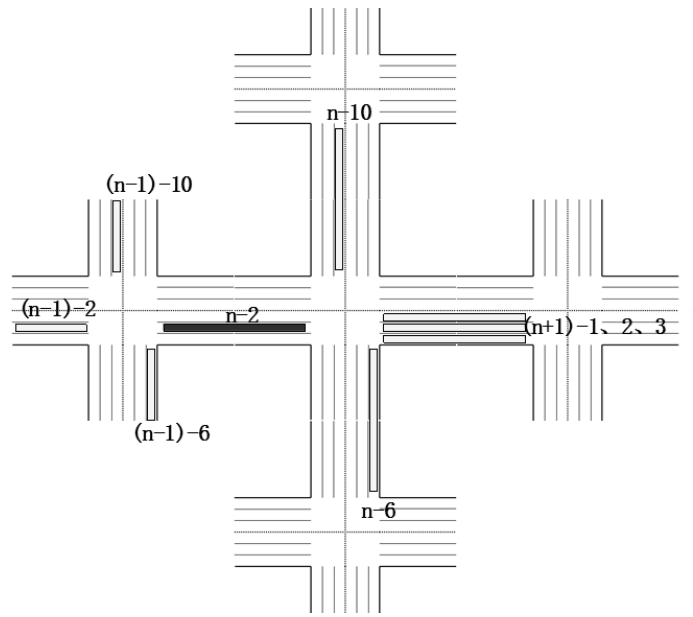

Figure 2. CA(intersection)MODULE

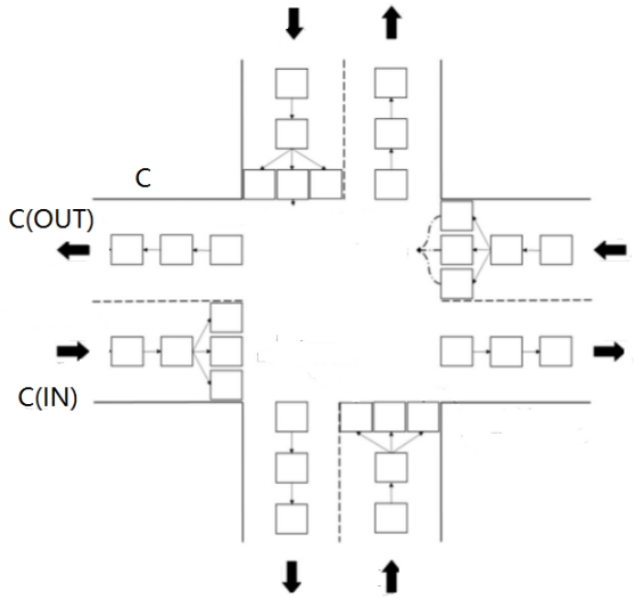

Figure 3. CA(lane)MODULE

Fig. 1 illustrates the basic lane module and parameters for it, we define every single vehicle or some vehicles linked into queue in the equations related to its current position and the time, which make us to calculate the queue with any time $t$ that is wanted, the queue length $1(t)$ is taken into the control equation and the prediction module that we do not study in this paper. Variable Q presents the flow value and variable $\rho$ presents the flow density.

Fig. 2 shows that how CA(intersection) module works, variable $n$ presents intersection number while $n-1, n+1$ means upstream and downstream. When the dark colour marked lane $n-2$ (intersection $\mathrm{n}$, lane 2) is called to judge whether to turn the current phase signal to next, the information of all 
those light color marked lane in fig. 2 will be collected by the control module. The parameter sets of lane $n-2$ are construction of all the lanes interacted which are marked.

The basic CA(intersection) module comes from Wei[8] with one lane per road and simple parameter, the basic control equation is built from reconstruction of N-S equation as follow:

$$
\frac{d u}{d t}=f_{b}-\frac{1}{\rho} \nabla p+\mu \nabla^{2} \mathrm{~V}
$$

$\frac{d u}{d t}$--inertia force, $f_{b}$--mass force, $-\frac{1}{\rho} \nabla p$--pressure gradient force, $\mu$--viscosity coefficient;

To use finite difference method to decompose equation(1) on $\mathrm{x}$ axis to get the control module:

$$
l(t)_{n, k}^{t+1}=\left(1-2 \lambda_{1}-\lambda_{2}\right) l(t)_{n, k}^{t}+\left(\lambda_{1}-\lambda_{2}\right) l(t)_{n-1, k}^{t}+\left(\lambda_{1}+\lambda_{2}\right) l(t)_{n+1, k}^{t}+\frac{\lambda_{2}}{2}
$$

With: $\lambda_{1}=\frac{\mu \Delta x}{(\Delta t)^{2}}, \lambda_{2}=\rho \mu \Delta t, \mathrm{k}$--signal phase

Fig. 3 explains that $\mathrm{CA}($ lane) module consist of 3 lanes per road, which enabled left turn, straight and right turn.

Here we define that each cell in the lane can be occupied with 3 vehicles also with each safe distance. The CA(lane) module provide the control system the dynamic characteristics of traffic flow at any moment $t$. The constraint for the vehicles and cells are reasonable when jam occurs do not talk in this paper.

\section{Rule optimization}

According to Fig 3, to maximize the traffic volume of each intersection represents that every cell $\mathrm{x}$ of $\mathrm{CA}($ lane) in each intersection counts sum of vehicles either put in or put out during interval $\mathrm{t}$.

$$
F(x)=M A X \sum_{\forall t \in T} \sum_{\forall i \in C_{I N}} x_{i}^{t}
$$

\section{1 flow distribution module}

Based on the flow distribution control rule, when any chosen lane corresponding to any phase is in the process of heading for the downstream lane, it will confluences with other associated with lanes related to same phase at downstream entrance of the local intersection. At the same time, its own traffic flow rate associated with adjacent lanes will determine the traffic allocation of upstream intersections. With different forms of injections at the entrance of each intersection different distribute rate will be chosen [9]:

$$
\sum_{i} M_{n} Q_{n}^{\text {out }}(t)=\left\{\begin{array}{c}
M_{n+1} Q_{n}^{\text {in }}\left(t, L_{n}\right) / M_{n} \\
M_{n+1} Q_{n}^{\max } / \mathrm{M}_{n} \\
\sum\left[\min \left(M_{n+1} Q_{n+1}^{\text {out }}\left(t, L_{n+1}\right)\right)\right] / \mathrm{M}_{n}
\end{array}\right.
$$

M--distribution coefficient related to $1(\mathrm{t})$

\section{2 optimization}


The parameter sets are defined with each initial value under Fig1.,when any thresholds of the lanes in the net are achieved by current pressure, the local control system aims to solve its own demand so that a green phase time $g_{p}$ is allocated and the start time $t_{g p}$ is arranged. With comparison of prediction superposition and current release of the characteristic pressure, the control system reassigns the matching rule parameters between linked input and output in their own range. $\alpha_{j}$--all the parameter sets.

$$
\begin{aligned}
& F\left(g_{p}\right)=\sum_{j-1}^{n} \alpha_{j}^{*} P_{j} \\
& P_{j}=F\left[\left(t_{j}^{g p}-t_{j+1}^{g p}\right),\left(t_{j}^{g p}+t_{j-1}^{g p}\right) L\right]
\end{aligned}
$$

This solution analyzes the changing process of the traffic flow by hydrodynamic. It basically explains how traffic flows can transfer continuously. In this way we can effectively characterize the instability of traffic states when they are at any of the critical points, and then return the parameters' change area. Hence, this basic rule designs the space for the self-organizing control system, which makes avoiding abrupt system concussion in a sudden.

\section{Simulation verification}

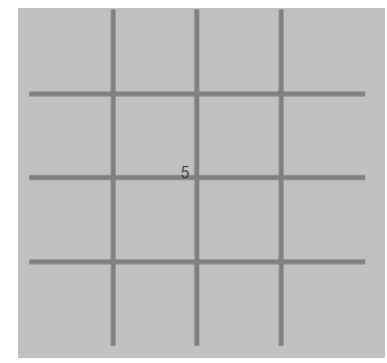

Figure 4. Road net of simulation

Fig.4 shows that the road net of simulation consist of 9 intersections which are linked as $\mathrm{CA}$ (intersection) module, 8 phases are chosen for all intersections. Maximum and minimum of signal cycle length are designed to $120 \mathrm{~s}$ and $60 \mathrm{~s}$; Maximum and minimum of vehicles' speed are designed to $60 \mathrm{KM} / \mathrm{H}$ and $8 \mathrm{KM} / \mathrm{H}$. The vehicles input into boundary from $600 \mathrm{puc} / \mathrm{h}$ to $1800 \mathrm{pcu} / \mathrm{h}$. The whole simulation lasts for 3600 s. Time step is defined as $15 \mathrm{~s}$, data analysis will start after vehicles have uniformly distributed in the network. The intersection 5 is chosen for observation.

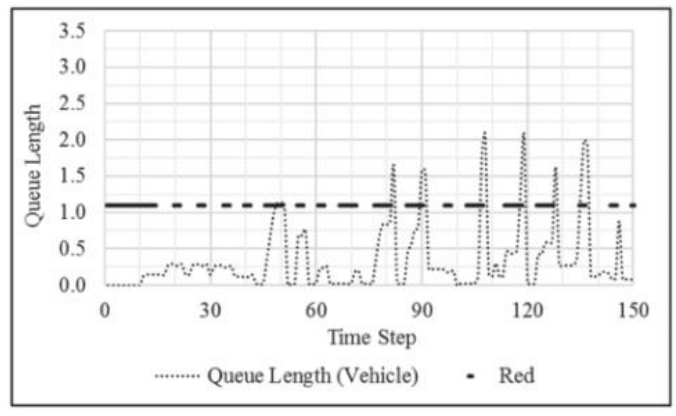

$\mathrm{a}$

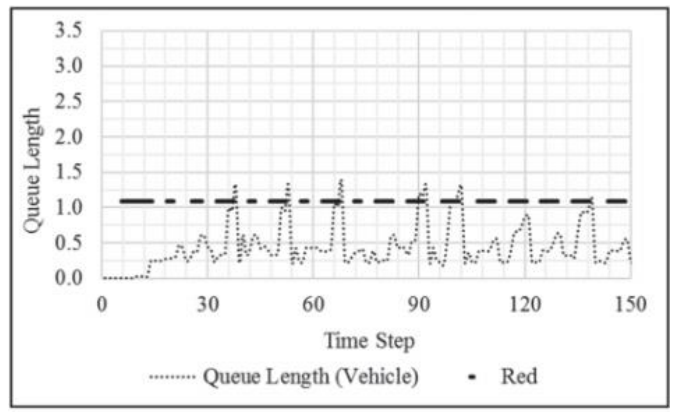

b 


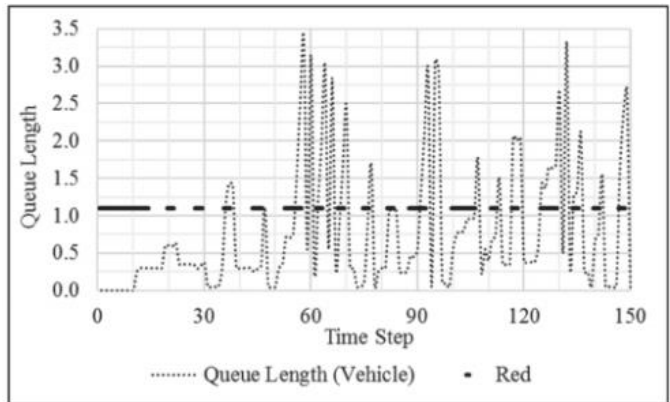

C

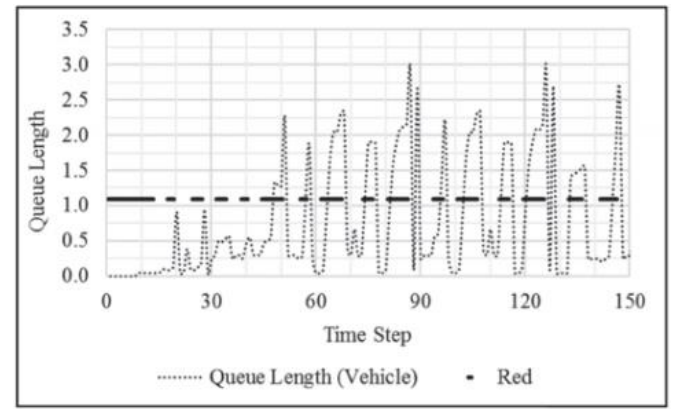

d

Figure 5. a.East to West without optimization b.East to West with optimization c. North to South without optimization b.North to South with optimization

Comparing with $\mathrm{a} / \mathrm{b}$ and $\mathrm{c} / \mathrm{d}$ in Fig.5,it is obviously that the self-organization signal control with rule optimization produce less traffic-jam than that without rule optimization, it also shows that after rule optimization the signal control system try to avoid jam in advance that is what self-organization works. The results confirms that the method really modify the evolution in a fast way to adapt to traffic flow's dynamic change.

\section{Conclusions}

This research has successfully demonstrated how to find different intersections' parameter sets and optimize them, which to satisfied their own maximum traffic volume as objective function. The new method can describe the dynamic characteristics by using the hydrodynamics models of traffic-flow, but also can create connections between interacted intersections under self-organization. The response speed of the signal control system is strengthened so that the signal control system can start to eliminate traffic-jam much earlier, the result is that each lane increases output vehicles during each phase no matter whether it is main road. By means of the computer simulation, the application of signal self-organizing control under hydrodynamic is proved effective in urban traffic. It provides comparison and reference for further solving such the complex system of traffic signal control.

\section{Acknowledgments}

This work is supported by the International Exchange Program for Graduate Students, Tongji University (No. 201801222).

\section{References}

1. M.J.Lighthill,G.B.Whithan,kinematic waves:2,229,317-345(1955)

2. T.Nagatani,Physi A, 261:599-607(1998)

3. Y.XUE,[D],ShangHai Unversity,21-29(2002)

4. A.K.Gupta,S.Sharma,P.Redhu,NonlinerDyn,80:1091-1108(2015)

5. O.M.Biham,A.Levine,Physi.E 46:6124-6127(1992)

6. C.Debashish,S.Andreas,Physi.E,59:1311-1314(1999)

7. J.HWei,A.L.Wang,N.C.Du,IEEE TVT,54:744-747(2005)

8. J.H.Wei,[D], Shanghai JiaoTong University 32-39(2005)

9. D.Helbing,S.Lammer,J.P.Lebacque, $O C D G, 239-274(2009)$ 